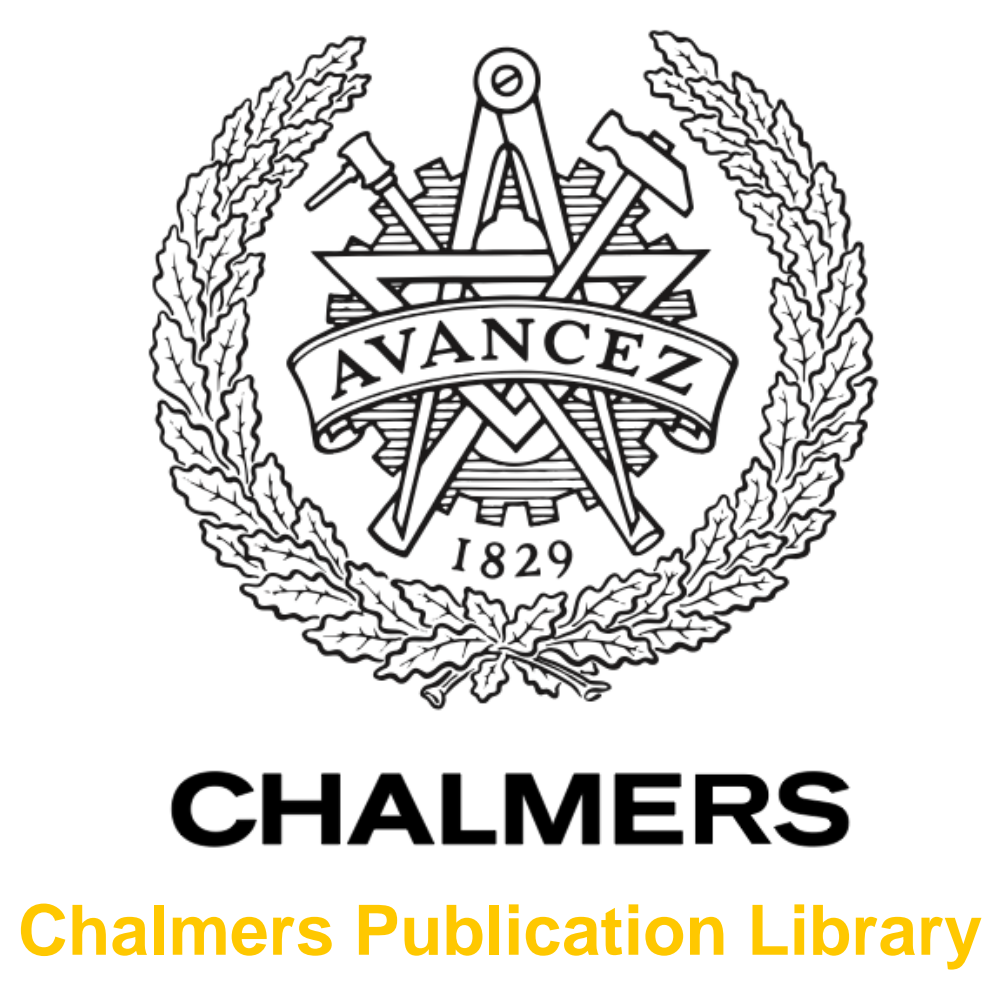

\title{
LTE MIMO throughput measurement method for characterization of multi-antenna terminal performance
}

This document has been downloaded from Chalmers Publication Library (CPL). It is the author's version of a work that was accepted for publication in:

2011 IEEE International Symposium on Antennas and Propagation, Spokane, USA, 3-8 July, 2011

Citation for the published paper:

Lötbäck Patané, C. ; Orlenius, C. (2011) "LTE MIMO throughput measurement method for characterization of multi-antenna terminal performance". 2011 IEEE International

Symposium on Antennas and Propagation, Spokane, USA, 3-8 July, 2011

Downloaded from: http://publications.lib.chalmers.se/publication/143614

Notice: Changes introduced as a result of publishing processes such as copy-editing and formatting may not be reflected in this document. For a definitive version of this work, please refer to the published source. Please note that access to the published version might require a subscription.

Chalmers Publication Library (CPL) offers the possibility of retrieving research publications produced at Chalmers University of Technology. It covers all types of publications: articles, dissertations, licentiate theses, masters theses, conference papers, reports etc. Since 2006 it is the official tool for Chalmers official publication statistics. To ensure that Chalmers research results are disseminated as widely as possible, an Open Access Policy has been adopted.

The CPL service is administrated and maintained by Chalmers Library. 


\title{
LTE MIMO Throughput Measurement Method for Characterization of Multi-Antenna Terminal Performance
}

\author{
Christian S. Lötbäck Patané \\ Charlie Orlenius \\ Bluetest AB \\ Gothenburg, Sweden \\ christian.lotback@bluetest.se
}

\begin{abstract}
This paper presents experiences and results from LTE MIMO throughput measurements of a USB dongle in a reverberation test system. The measurements are performed with the dongle itself and also when it is connected to external diversity antennas with high and low correlation. Three different maximum bit rates are used, 59, 70 and 81 Mbps. The results illustrate that it is possible to separate a good antenna design from a bad from reverberation chamber measurements. Also, the effect of using a laptop test fixture, or a laptop dummy, instead of having the host computer present in the chamber, is demonstrated. It is shown that the noise induced by the host computer degrades the performance of the dongle.
\end{abstract}

Keywords - laptop test fixture; throughput; LTE; MIMO; reverberation chamber;

\section{INTRODUCTION}

Long-term evolution (LTE) multiple-input-multipleoutput (MIMO) communication systems are becoming more widespread and common for each year. Implementing the LTE MIMO technology in cellular phones and wireless modems (dongles) increases the data rates and the quality of the service. Therefore, there is an increased demand for accurate and repeatable ways of characterizing these systems. The test methods must be able to separate between a device with a good and a bad performance. The reverberation chamber is one of the methods proposed, since it offers an efficient and easy measurement procedure to do so.

A reverberation chamber is a metal cavity with electromagnetic reflective walls. By injecting an electromagnetic field in the chamber, and by stirring that field, the chamber can be used to generate a 3-dimensional Rayleigh fading environment [1] with an exponential decaying power delay profile. The signal received by a device placed in the chamber is similar to the faded signal received by the end-user equipment in a real multipath propagation environment, like urban and indoor environments [2]. In contrast to the real world, the chamber will be a repeatable facility. Because of this, the reverberation chamber has become an established tool for analysis and characterization of various antenna parameters, such as radiation efficiency, impedance mismatch, diversity gain, MIMO antenna capacity, total radiated power (TRP), and total isotropic sensitivity (TIS). These measurement setups are well described in previous publications [3-6].

As LTE MIMO enabled test equipment and devices recently have become available, it has been possible to start the evaluation of the different test methods proposed for the LTE MIMO technology. This paper presents experiences from LTE MIMO throughput measurements performed in a Bluetest reverberation test system (Fig. 1). The measurements are performed with a USB dongle for three different maximum bit rates, 59, 70 and $81 \mathrm{Mbps}$. The dongle has connectors that are used to connect external diversity antennas with high and low correlation. Using this setup it is possible to see if a device with a good antenna design can be separated from a device with a bad antenna design, using the reverberation chamber.

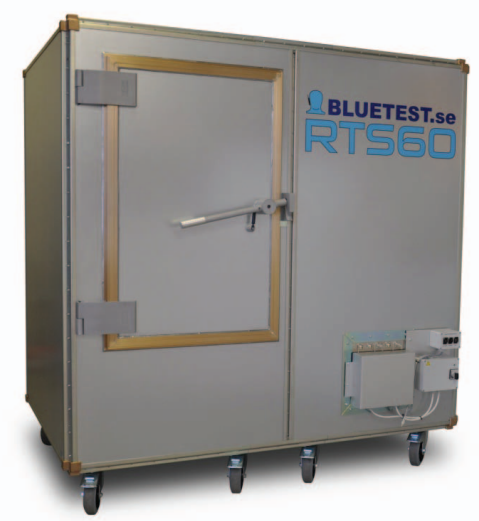

Figure 1. Photo of a Bluetest reverberation test system RTS60 used in the presented measurements. Cavity size of the chamber is $1.75 \mathrm{~m} \times 1.8 \mathrm{~m} \mathrm{x}$ $1.2 \mathrm{~m}$. 
One issue that arises when measuring the performance of USB dongles is the interference from the host computer. The amount of interference from the computer will depend on the laptop brand, model or even the specific unit at hand. A computer working with maximum performance will also give more noise than a computer in power-saving mode. Thus the measured performance of the dongle will be dependent on the specific host computer used and will not be the same if measured in different labs. One way to solve this problem is to use a so called laptop test fixture (LTF). This is basically a laptop dummy, which removes the interference from the host computer but works to keep the parts of the computer that are important for the optimized performance of the dongle (ground plane, mother board, etc.) intact. In this paper the effect of using such an LTF will be demonstrated.

\section{Measurement Procedure}

The measurements were performed in a Bluetest reverberation test system, series 6 (RTS60). This is a modestirred reverberation chamber, equipped with three transmitting antennas and other hardware and software that enable MIMO measurements. The chamber is also equipped with an optical USB interface that makes it possible to perform measurements without the host computer present in the chamber. There was no load in the chamber during the measurements and thus the delay spread was approximately 200 ns. Furthermore, an LTE enabled communication tester (CMW500 from Rhode \& Schwarz) was connected to the RTS60. At the time of testing, this communication tester supported LTE MIMO transmission mode 3: Open loop spatial multiplexing. Fig. 2 shows a schematic picture of the measurement setup.

The throughput was measured for a fix output power and a continuous stirrer sequence with a sequence length of 30 seconds (50 samples á 500 frames). The measurements were performed on LTE band 7, downlink channel 2850 (2630 $\mathrm{MHz}$ ) with a bandwidth of $20 \mathrm{MHz}$. More settings can be studied in Table I.

The dongle used in the measurements was a Samsung GT-B3730 and the host computer was an HP ProBook 6550b. In most of the measurements the host computer was placed outside the chamber and the dongle was connected to it via the chamber optical interface. Measurements were also performed with the computer present in the chamber and with a laptop test fixture (LTF). In the latter case the dongle was connected to the LTF, which in turn was connected to the optical interface (see Fig. 3). The LTF was an IBM 600E, where one of the USB ports was connected to an external USB cable and further connected to the USB interface in the chamber. The internal electronics of the LTF was disabled to avoid disturbing noise.

Moreover, the dongle has connectors that enable external antennas to be attached to it. These were used for connecting external multiport antennas with high correlation $(>0.9)$ and low correlation $(<0.1)$. The external antennas have been designed by Sony Ericsson to be used as MIMO test phones and can be studied in Fig. 4. The low correlation antenna has two miniature ceramic BT/WLAN antennas put on each long-side of the pcb. These are from Pulse, type W3008c. For the high correlation a dual feed PIFA was used. The antennas were also used in previously presented measurements performed by NTT DOCOMO in [7], where additional antenna data can be found. Using this setup it was possible to see if a dongle with a good antenna design could be separated from that with a bad design from measurements in a reverberation chamber.

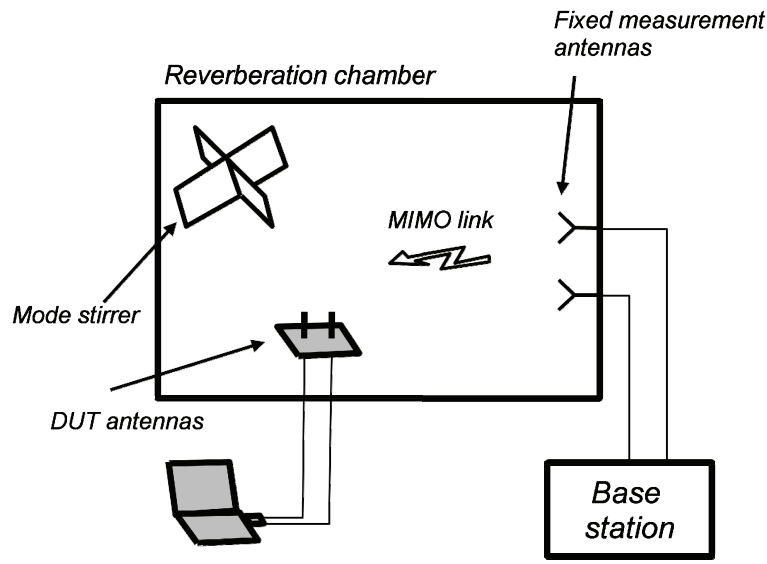

Figure 2. Schematic picture of the measurement setup for the LTE MIMO measurements in the RTS60.

TABLE I. THE SETTINGS USED FOR THE LTE MIMO MEASUREMENTS.

\begin{tabular}{|l|l|}
\hline \multicolumn{1}{|c|}{ Parameter } & \multicolumn{1}{c|}{ Value } \\
\hline Downlink channel & $2850(2630 \mathrm{MHz})$ \\
\hline Bandwidth & $20 \mathrm{MHz}$ \\
\hline Modulation & $64-\mathrm{QAM}$ \\
\hline Frames per sample & 500 \\
\hline $\begin{array}{l}\text { Number of downlink resource } \\
\text { blocks }\end{array}$ & 96 \\
\hline Transport block size index & $\begin{array}{l}15,17 \text { and } 19 \quad(=59,70 \text { and } 81 \\
\text { Mbps })\end{array}$ \\
\hline Connection type & User defined \\
\hline Transmission mode & Open loop spatial multiplexing \\
\hline
\end{tabular}




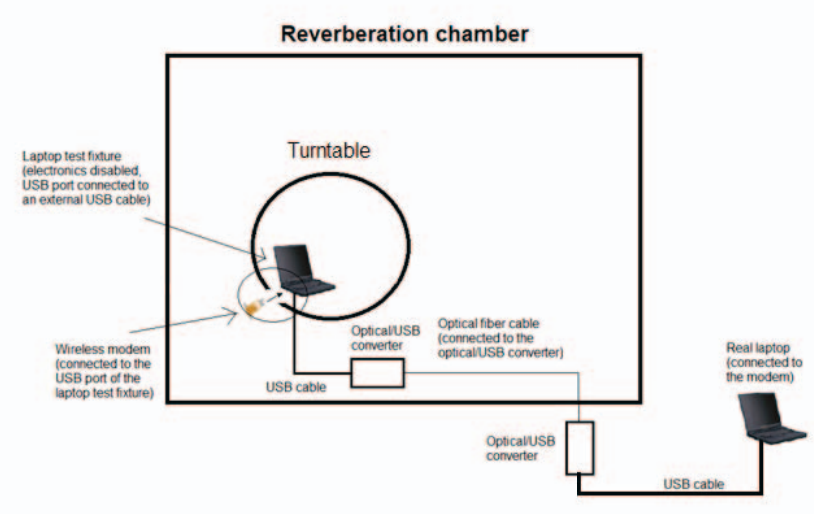

Figure 3. A schematic picture of the measurement setup for measuring throughput with a laptop test fixture connected to the USB interface in the RTS60.

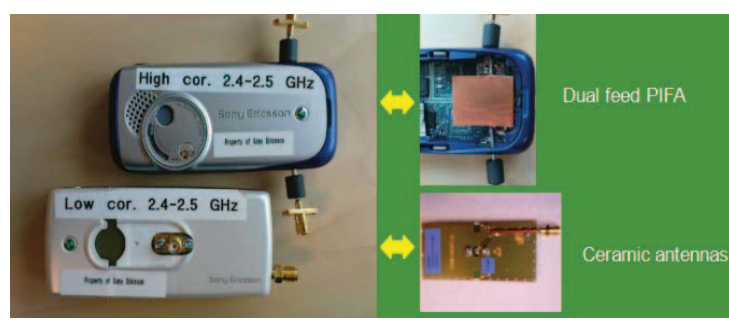

Figure 4. The external antennas with high and low correlation used in the measurements.

\section{RESULTS}

This section presents the results of the LTE MIMO measurements in the Bluetest RTS60 system.

\section{A. LTE MIMO measurements using different bit rates and external multiport antennas}

Fig. 5 to Fig. 7 shows the results from the measurements using the external connected multiport antennas for three different bit rates; 59, 70 and 81 Mbps. As can be seen in the figures, the dongle always reaches the maximum throughput at higher power values. The throughput value is dependent on the external antenna used. The external antenna with high correlation gives a lower throughput for the same power value compared to the antenna with a low correlation. This is expected and shows that it is possible to separate a dongle with a good design from that with a bad design.

The figure also shows that the device is detaching when the throughput is about $40-60 \%$ of the maximum bit rate. This might be due to an instable dongle or the instability of the communication tester. Regardless of the reason, this limits the throughput values that can be measured and thus the characterization of the dongle.

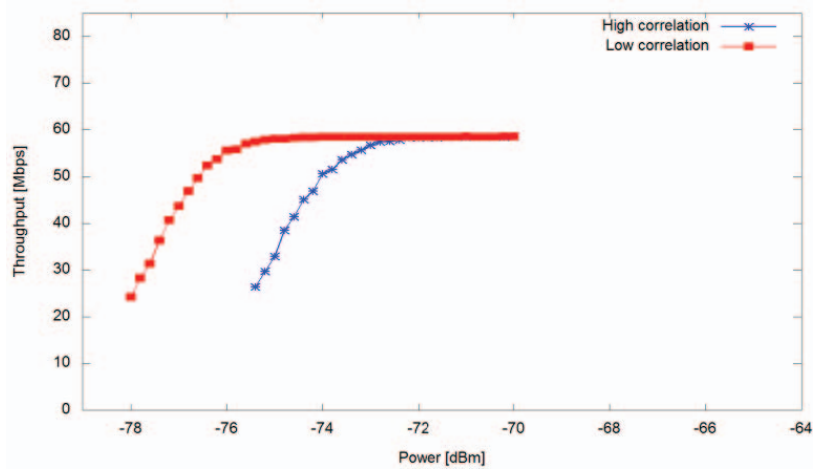

Figure 5. Results from the LTE MIMO throughput measurements with a USB dongle in the Bluetest RTS60, using a maximum bit rate of $59 \mathrm{Mbps}$ and two different multiport antennas. The setup with the high correlation antenna has worse performance than the setup with the low correlation antenna.

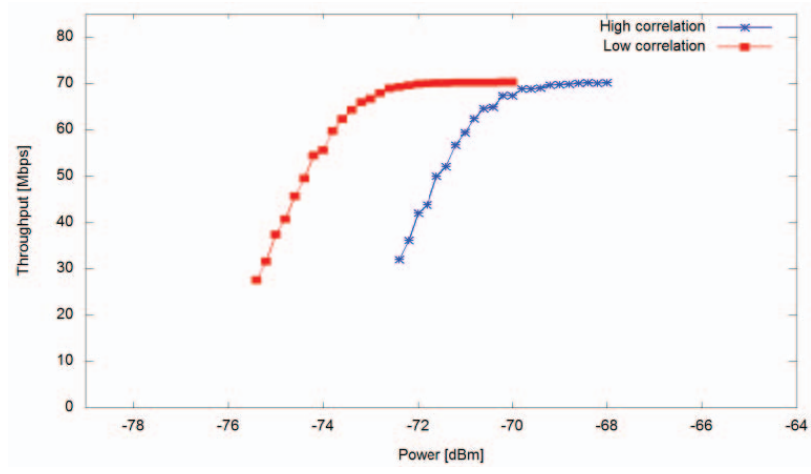

Figure 6. Results from the LTE MIMO throughput measurements with a USB dongle in the Bluetest RTS60 using a maximum bit rate of $70 \mathrm{Mbps}$ and two different multiport antennas. The setup with the high correlation antenna has worse performance than the setup with the low correlation antenna.

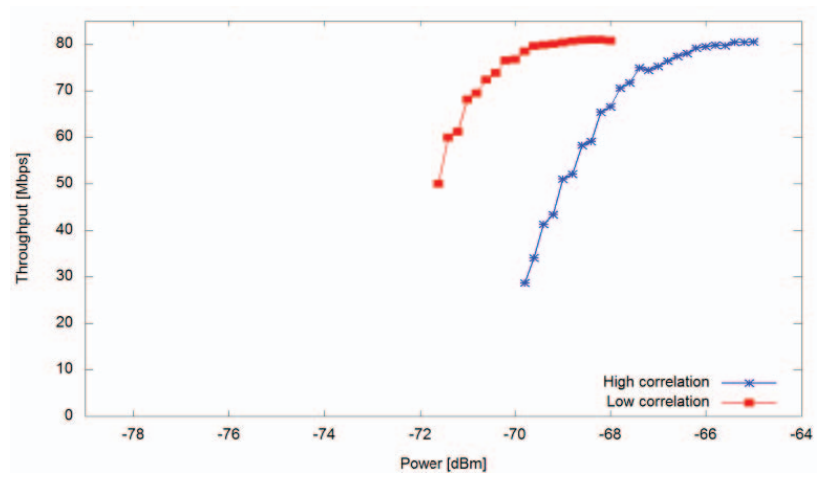

Figure 7. Results from the LTE MIMO throughput measurements with a USB dongle in the Bluetest RTS60 using a maximum bit rate of $81 \mathrm{Mbps}$ and two different multiport antennas. The setup with the high correlation antenna has worse performance than the setup with the low correlation antenna. 


\section{B. Repeatability}

Fig. 8 shows the results from three and two repeatable throughput measurements with a maximum bit rate of 70 Mbps and $81 \mathrm{Mbps}$, respectively. For the first data rate the measurements were performed with the dongle connected to the LTF. In this case the LTF was removed and replaced in the chamber at approximately the same place. For the second data rate the dongle was connected to the external multiport antenna with low correlation. In this case the measurements were performed in different days, however without anything moved in the chamber. The same settings were used for the repeated measurements. It is clearly seen that the result is repeatable for both data rates.

\section{Reducing noise impairment by using a laptop test fixture}

A comparison of the throughput values was also performed between connecting the dongle to an LTF and directly to the host computer present in the chamber. The maximum bit rate was $81 \mathrm{Mbps}$ and the result can be studied in Fig. 9. The performance is seen to be about $2 \mathrm{~dB}$ worse with the host computer present and activated in the chamber. This indicates that the interference from the computer significantly affects the performance of the dongle.

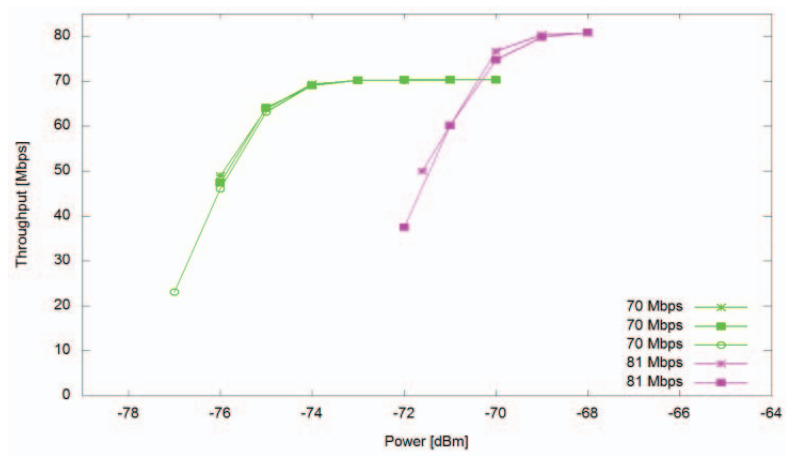

Figure 8. Results from three consecutive LTE MIMO throughput measurements with a bit rate of 71 and $81 \mathrm{Mbps}$. The dongle is connected to the laptop test fixture. The result is repeatable.

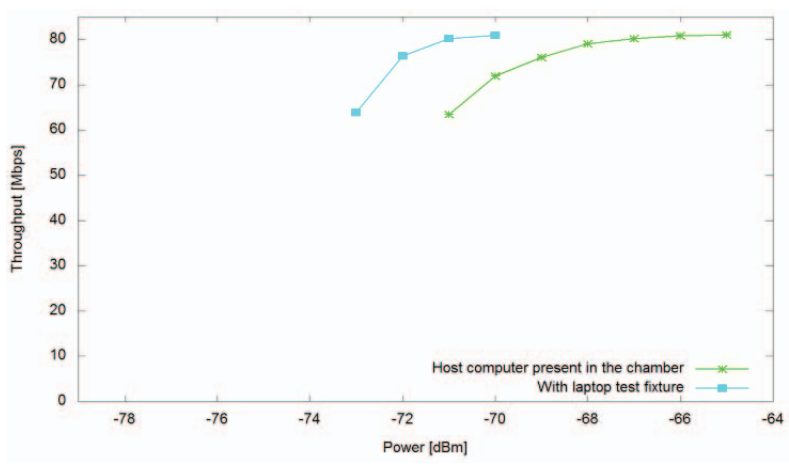

Figure 9. Comparison of the LTE MIMO throughput between connecting the dongle to a laptop test fixture and to a host computer present in the chamber. The maximum bit rate was $81 \mathrm{Mbps}$. The performance is approximately $2 \mathrm{~dB}$ worse with the host computer present in the chamber.

\section{CONCLUSIONS}

LTE MIMO measurements of a USB dongle has been performed in a Bluetest reverberation test system. Maximum bit rate was reached for all tested modulation and coding schemes. The measurements show that it is possible to see differences in performance when using different bit rates and to separate a dongle with a good antenna design from that with a bad design. Also, the results are repeatable. Finally, it is shown that the presence of the host computer in the chamber degrades the performance of the dongle compared to using a laptop test fixture.

\section{ACKNOWLEDGMENT}

This work has been carried out in the Chase VINN Excellence Center at Chalmers University of Technology, Gothenburg, Sweden.

\section{REFERENCES}

[1] J. G. Kostas, B. Boverie, "Statistical model for a mode-stirred reverberation chamber", IEEE Transactions on Electromagnetic Compatibility, Vol. 33, No. 4, Nov. 1991.

[2] D. M. Pozar, "Microwave and RF design of wireless systems", John Wiley and sons INC, 2001.

[3] M. Andersson, A. Wolfgang, C. Orlenius, J. Carlsson, "Measuring Performance of 3GPP LTE Terminals and Small Base Stations in Reverberation Chambers", Chapter 12 of "Long Term Evolution: 3GPP LTE Radio and Cellular Technology", CRC Press, 2009.

[4] P-S Kildal, "Overview of 6 Years R\&D on Characterizing Wireless Devices in Rayleigh Fading Using Reverberation Chambers", International Workshop on Antenna Technology (iWAT07), Cambridge, UK, pp. 162-165, 2007.

[5] K. Rosengren and P.-S. Kildal, "Radiation efficiency, correlation, diversity gain, and capacity of a six monopole antenna array for a MIMO system: Theory, simulation and measurement in reverberation chamber", Proceedings IEE, Microwave Antennas Propagation, vol. 152, no. 1, pp.7-16, February 2005. See also Erratum published in August 2006.

[6] P.-S. Kildal and K. Rosengren, "Correlation and capacity of MIMO systems and mutual coupling, radiation efficiency and diversity gain of their antennas: Simulations and measurements in reverberation chamber", IEEE Communications Magazine, vol. 42, no. 12, pp. $102-$ 112, Dec. 2004

[7] M. Franzén, C. Orlenius, D. Kurita, Y. Okano, " Comparison of MIMO OTA Performance characteristics as Measured in Reverberation Chamber and Spatial Fading Emulator", COST 2100 TD(10)10084, Athens, Greece, Feb. 2010. 